\title{
Formation of Massive Stars by Growing Accretion
}

\author{
André Maeder \\ Geneva Observatory, CH-1290 Sauverny, Switzerland
}

\begin{abstract}
We calculate pre-Main Sequence evolutionary tracks with accretion rates growing with the actual stellar masses. We show that accretion rates growing at least as $M^{1.5}$ are necessary to fit the constraints on the lifetimes and HR diagram. Most interestingly, such accretion rates growing with the stellar mass well correspond to those derived from observations of mass outflows (Churchwell 2000; Henning et al. 2000). These rates also lie in the permitted region of the dynamical models.
\end{abstract}

The formation of massive stars is one of the last frontiers in stellar evolution. There are three scenarios in literature. 1). The classical scenario of pre-MS evolution at constant mass. 2). The coalescence scenario (cf. Bonnell et al. 1998). It was proposed due to the alleged difficulty of forming massive stars by accretion, because of radiation pressure effects. However, as discussed below, there is a domain of dynamically possible accretion rates. Recently, Elmegreen (2000) has shown that the star density in young clusters is probably not high enough for the collision scenario to operate. He emphasizes that there is not enough time for a protostar to move around in a young cluster and to coalesce with other stars. 3). The scenario of massive star formation by accretion (Beech and Mitalas 1994; Bernasconi and Maeder 1996). We show here that the accretion scenario is possible, if the accretion rates $\dot{M}_{\text {accr }}$ grow fast enough with the already accreted stellar mass.

Firstly, we recall that there is a basic difference between low and high mass stars. For high mass stars (mass $\geq 8 M_{\odot}$ ), the accretion times $t_{\text {accr }}$ are longer than the Kelvin-Helmholtz time $t_{\mathrm{KH}}$, thus the stars ignite their nuclear reactions and set on the ZAMS, still accreting and hidden in their molecular clouds. For low mass stars, $t_{\mathrm{accr}} \leq t_{\mathrm{KH}}$ is the rule.

Models with constant accretion rates or slowly growing accretion rates lead to much too long pre-MS lifetimes. Also the birth lines, which is the path in the HR diagram followed by accreting stars, do not provide a good upper envelope of the observations. Norberg and Maeder (2000) have calculated several grids of pre-MS tracks with accretion rates growing like $\dot{M}_{\text {accr }}=\dot{M}_{\text {ref }}\left(\frac{M}{M_{\odot}}\right)^{\varphi}$, with values of $\varphi$ equal to $0.5,1.0$ and 1.5 and also for different values of $\dot{M}_{\text {ref }}$. The best fit to the short lifetimes required $\left(t \leq 10^{6} \mathrm{yr}\right.$, cf. Elmegreen, 2000) and to the observations of PMS stars in the HR diagram is achieved for $\varphi \simeq 1.5$ and for $\dot{M}_{\text {ref }}$ of the order of $10^{-5} M_{\odot} \mathrm{yr}^{-1}$, (cf. Fig. 1).

Temperatures, luminosities and outflow rates of UC HII regions have been studied by radio and IR observations by Churchwell (2000) and Henning et al. (2000). Huge bipolar outflows have been observed. Remarkably, the outflow 


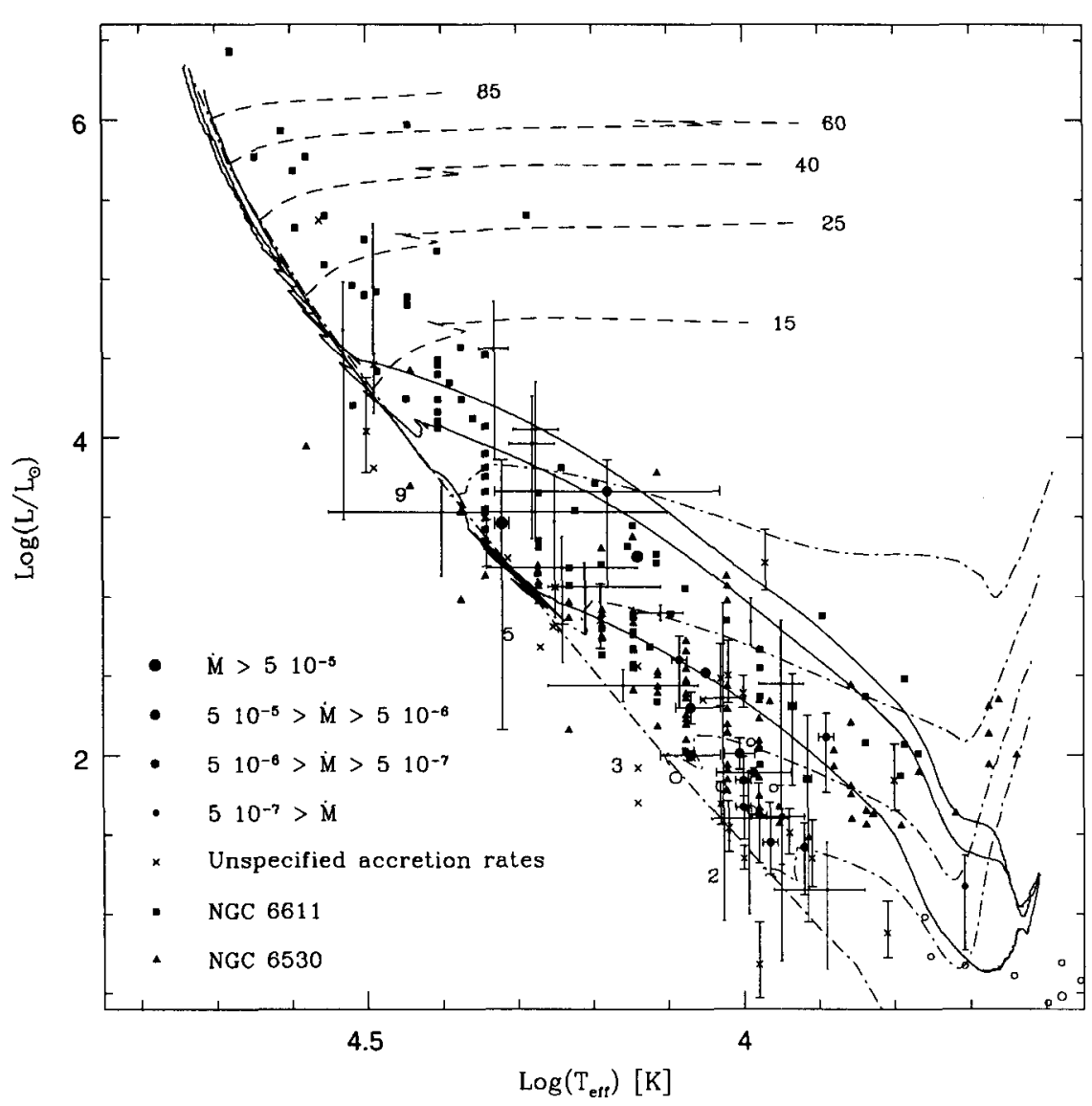

Figure 1. The continuous lines represent birthlines obtained with $\varphi$ $=1.5$ and $\dot{M}_{\text {ref }}$ being equal to $10^{-6}, 5 \cdot 10^{-6}$ and $10^{-5} M_{\odot} \mathrm{yr}^{-1}$ from bottom to top respectively. The points represent observations from various sources collected by Norberg and Maeder (2000). The broken lines are post-MS tracks, and the dot-broken lines pre-MS tracks with constant mass. 
rates $\dot{M}_{\text {out }}$ behave continuously like $L_{\text {bol }}^{0.7}$ over 6 decades of luminosity. At solar luminosity, the values of $\dot{M}_{\text {out }}$ are about $10^{-5} M_{\odot} \mathrm{yr}^{-1}$, i.e. of the same order as the currently estimated accretion rates. However for massive stars with $L$ between $10^{4}$ and $10^{6} L_{\odot}$, the outflow rates $\dot{M}_{\text {out }}$ are in the range of $10^{-3}$ to $10^{-2} M_{\odot} \mathrm{yr}^{-1}$. From the masses present in the outflows and the luminosity of the central object, Churchwell estimates that the fraction $f$ of the infalling material incorporated into the star is about $15 \%$, while $85 \%$ is deflected in the outflows. With a mass-luminosity relation appropriate for the interval of 2 to $85 M_{\odot}$, we get

$$
\dot{M}_{\text {accr }}=1.510^{-5} \frac{f}{1-f}\left(\frac{M}{M_{\odot}}\right)^{1.54} \quad \mathrm{M}_{\odot} \mathrm{yr}^{-1},
$$

where $f$ is the accreted fraction of the infalling material. It is remarkable that the slope and constant factor derived in Fig. 1 are so close to that obtained from UC HII regions.

Not all values of $\dot{M}_{\text {accr }}$ are possible (cf. Wolfire and Cassinelli 1987). For too low $\dot{M}_{\text {accr }}$, the momentum in the radiation is larger than that of the wind, so accretion is not possible, while for too high $\dot{M}_{\text {accr }}$, the shock luminosity created by accretion becomes supra-Eddington. The rates given by Eq. (1) are in the permitted domain, even when rotation and accretion in the midplane are accounted for (cf. Nakano, 1989).

In the literature, an expression like $\dot{M}_{\text {accr }} \simeq \frac{v_{\text {sound }}^{3} \text { is often found. According }}{G}$ to it, high accretion rates as given by Eq. (1) for massive stars would imply a relatively high temperature $T$ of the infalling gas. However, high $T$ are not observed (Caselli and Myers 1995), and strong turbulent motions, for which there are many evidences, may well provide the necessary initial support of the cloud. Moreover, turbulent motions fed by the stellar radiation may critically participate to the regulation of the accretion rates of the matter falling from the cloud onto the disk.

\section{References}

Beech, M, Mitalas, R. 1994, ApJS, 95, 517

Bernasconi, P.A., Maeder, A. 1996, A\&A, 307, 829

Bonnell, I.A., Bate, M.R., Zinnecker, H. 1998, MNRAS, 298, 93

Caselli, P., Myers, P.C. 1995, ApJ, 446, 665

Churchwell, E. 2000 in Unsolved Problems in Stellar Evolution, Space Telescope.

Sci. Inst. Symp. Ser., vol 12, Ed. M. Livio, Cambridge Univ. Press, 41.

Elmegreen, B. 2000, astro-ph/0005189

Henning, Th., Schreyer, K., Launhardt, R., et al. 2000, A\&A, 353, 211

Nakano, T. 1989, ApJ, 345, 464

Norberg, P., Maeder, A. 2000, A\&A, 359, 1025

Wolfire, M.G., Cassinelli, J.P. 1987, ApJ, 319, 850 